\title{
Neural Network Effectiveness Evaluation While the Intersection of Images in the Receptor Field
}

\author{
SIRANUSH SARGSYAN \\ Department of Programming and Information Technologies \\ Yerevan State University \\ A.Manoogian, 1, Yerevan, 0025 \\ ARMENIA \\ ANNA HOVAKIMYAN \\ Department of Programming and Information Technologies \\ Yerevan State University \\ A.Manoogian, 1, Yerevan, 0025 \\ ARMENIA
}

\begin{abstract}
The study and application of neural networks is one of the main areas in the field of artificial intelligence. The effectiveness of the neural network depends significantly on both its architecture and the structure of the training set. This paper proposes a probabilistic approach to evaluate the effectiveness of the neural network if the images intersect in the receptor field. A theorem and its corollaries are proved, which are consistent with the results obtained by a different path for a perceptron-type neural network.
\end{abstract}

Key-Words: - neural network, receptor field, training sequence, exciting and inhibiting connections, probability of excitation, intersection of images

Received: May 22, 2020. Revised: November 14, 2020. Accepted: December 5, 2020. Published: December 16, 2020.

\section{Introduction}

A continuous stream of sensory information from the outside world is processed in the human brain. Understanding the human memory process is a serious problem; new images are remembered in such a form that the previously memorized ones are not modified or forgotten. This creates a dilemma: how the memory remains plastic, capable of perceiving new images, and at the same time maintains stability, ensuring that the images are not destroyed or damaged in the process of functioning[1-4] .

The traditional artificial neural networks were unable to solve the problem of stability-plasticity. Very often neural network training on a new image destroys or alters the results of previous training. Training images are passed into the network to accumulate information about the belonging of these images to the corresponding classes. In networks with back propagation, for example, training images (vectors) are fed to the network input sequentially until the network has been trained on the entire input set. If however a fully trained network needs to memorize a new training vector, it can change the weights so much that it requires a complete retraining of the network.

Many works are devoted to the selection of training images supplied to the input of the network, as well as to the location of these images in the receptor field when they are consistently fed to the input of the network. Different teaching methods and related research and evaluation of network parameters have the goal of improving the efficiency of the neural network, i.e. increasing the probability of excitation of layers of the neural network where the transmitting information is accumulated [2,3].

In the present paper we propose a method for studying the probability of correct classification of input data in the case of the intersection of these images in the receptor field when they are passed to the network. It turns out that the efficiency of the neural network can be improved by reducing the area of intersection of the input images during training. It can be said that in a certain sense, the problem of stability-plasticity for artificial neural networks is solved by choosing the structure of the 
training set and the parameters of the classification model during training.

In the paper the theorem and its corollaries are proved where the obtained results coincide with the results proved for a perceptron-type neural network [3].

\section{Problem Statement and Solution}

A direct propagation neural network is considered, which consists of a layer $\mathrm{S}$ of input nodes (Selements), hidden layers with associative Aelements and an output layer with reactive elements $[3,6,7]$. Denote $N_{S}$ the number of S-elements and $N_{A}$ the number of A-elements.

Neurons have unidirectional connections, do not contain connections between the elements inside the layer and feedback connections between the layers. The neurons of the input layer are connected to the neurons of the hidden layer by excitatory and inhibitory connections in a random way. The outputs of all the neurons of the hidden layer are connected to the neurons of the output layer $[2,3]$.

An image is formed in the receptor field, corresponding to external irritation. Under the image we mean a certain vector, the coordinates of which correspond to individual elements of the receptor field and can take the values 1 and 0 , depending on whether the corresponding element is excited or not.

Thus, the image $\mathrm{X}$ on the retina (receptor field) is represented by the vector $X=\left\{x_{j}\right\}$, $\left(j=1,2, \ldots, N_{s}\right)$, each component of which can be in an excited or unexcited state:

$$
x_{j}=\left\{\begin{array}{l}
1, \quad \text { if the element is excited } \\
0, \quad \text { if the element is not excited }
\end{array}\right.
$$

Below we prove a theorem that gives the condition for the synthesis of an effective structure of a neural network for practical application.

Theorem. Let A - element has a threshold, $n$ excitatory and $m$ inhibitory connections, randomly and equiprobably distributed on the retina.

If the images $X^{\prime}$ and $X^{\prime \prime}$ excite the retina, respectively, with success probability $p_{1}$ and $p_{2}$, and with failure probability $q_{1}=$ $1-p_{1}, q_{2}=1-p_{2}$, then the probability that the A- element is excited from each $X$ 'and $X^{\prime \prime}$ is

$$
P_{12}=\sqrt{\frac{P_{1}}{p_{1}} \frac{P_{2}}{p_{2}} \frac{Q_{1}}{q_{1}} \frac{Q_{2}}{q_{2}}}\left(p_{12}-p_{1} p_{2}\right)+P_{1} P_{2}
$$

where $P_{1}$ is the probability of A-element excitation when an image $X^{\prime}$ appears on the retina of neural network, $\quad P_{2}$ is the probability of A-element excitation when the image $X^{\prime \prime} \quad$ appears on the retina, and $P_{12}$ is the probability of A-element excitation when a pair of images $X^{\prime}$ and $X^{\prime \prime}$ from the both classes appear on the retina (the image $X^{\prime}$ is from the first class and the image $X^{\prime \prime}$ is from the second class), $Q_{1}=1-P_{1}, Q_{2}=1-P_{2}$.

Proof. Let the images $X^{\prime}$ and $X^{\prime \prime}$ excite the retina with probabilities $p_{1}$ and $p_{2}$, respectively. Since the state of each their component (being excited or unexcited) is random, so $X^{\prime}$ and $X^{\prime \prime}$ can be considered as random variables of $N_{S}$ dimension.

$X^{\prime}=\left\{x_{1}^{\prime}, x_{2}^{\prime}, \ldots, x_{N_{S}}^{\prime}\right\}, X^{\prime \prime}=\left\{x_{1}^{\prime \prime}, x_{2}^{\prime \prime}, \ldots, x_{N_{S}}^{\prime \prime}\right\}$

Let the random variable $X^{\prime}$ takes the value 1 with the probability $p_{1}$, and the value 0 with the probability $q_{1}=1-p_{1}$, and the random variable $X^{\prime \prime}$ takes the value 1 with the probability $p_{2}$, and the value 0 with the probability $q_{2}=1-p_{2}$.

The correlation coefficient $r_{s}$ between the random variables $X^{\prime}$ and $X^{\prime \prime}$ is determined by the formula

$$
r_{s}=\frac{\operatorname{CoV}\left(\mathrm{X}^{\prime}, \mathrm{X}^{\prime \prime}\right)}{\sqrt{\sigma\left(\mathrm{X}^{\prime}\right) \sigma\left(\mathrm{X}^{\prime \prime}\right)}}
$$

where $\operatorname{COV}\left(X^{\prime}, X^{\prime \prime}\right)=M\left(X^{\prime} X^{\prime \prime}\right)-M\left(X^{\prime}\right)$ * $M\left(X^{\prime \prime}\right)$, where $M\left(X^{\prime}\right)$ and $M\left(X^{\prime \prime}\right)$ are the mathematical expectations for $X^{\prime}$ and $X^{\prime \prime}$.

$$
M\left(\mathrm{X}^{\prime}\right)=\sum_{i=1}^{N_{S}} M\left(x_{i}^{\prime}\right) \sum_{\substack{i=1 \\ N_{S}}}^{N_{i}} 1 * P\left(x_{i}^{\prime}=1\right)+0
$$




$$
M\left(\mathrm{X}^{\prime \prime}\right)=\sum_{i=1}^{N_{S}} M\left(x_{i}^{\prime \prime}\right) \sum_{\substack{i=1 \\ N_{S}}}^{N^{\prime}} 1 * P\left(x_{i}^{\prime \prime}=1\right)+0
$$

All elements of the image $X^{\prime}$ are excited equally likely i.e.

$$
\begin{gathered}
P\left(x_{i}^{\prime}=1\right)=P\left(x_{i}^{\prime}=0\right)=P^{\prime} \text { and } \\
M\left(X^{\prime}\right)=N_{S} * P^{\prime}=p_{1}
\end{gathered}
$$

Similarly $M\left(X^{\prime \prime}\right)=N_{S} * P^{\prime \prime}=p_{2}$.

Define the vector $X^{\prime \prime \prime}=\left(X^{\prime} X^{\prime \prime}\right)$ as follows

$X^{\prime \prime \prime}=\left\{x_{1}^{\prime} x_{1}^{\prime \prime}, x_{2}^{\prime} x_{2}^{\prime \prime}, \ldots, x_{N_{S}}^{\prime} x_{N_{S}}^{\prime}\right\}$, where

$$
x_{i}^{\prime \prime \prime}=x_{i}^{\prime} x_{i}^{\prime \prime}=\left\{\begin{array}{c}
1, \quad \text { if } x_{i}^{\prime}=x_{i}^{\prime \prime}=1 \\
0, \text { otherwise }
\end{array}\right.
$$

$X^{\prime \prime \prime}$ is a random variable of $N_{S}$ dimension and

$$
\begin{aligned}
& M\left(\mathrm{X}^{\prime \prime \prime}\right)=\sum_{i=1}^{N_{S}} M\left(x_{i}^{\prime \prime \prime}\right)= \\
& \sum_{i=1}^{N_{S}} 1 * P\left(x_{i}^{\prime \prime \prime}=1\right)+0 * P\left(x_{i}^{\prime \prime \prime}=0\right)
\end{aligned}
$$

Suppose that all values of the coordinates of the vector $X^{\prime \prime \prime}$ are equiprobable i.e.

$P\left(x_{i}^{\prime \prime \prime}=1\right)=P\left(x_{i}^{\prime \prime \prime}=0\right)=P\left(i=1,2, \ldots, N_{S}\right)$.

So, $M\left(X^{\prime \prime \prime}\right)=N_{S} * P=p_{12}$.

The covariance of the random variables $X^{\prime}$ and $X^{\prime \prime}$ is expressed by the formula

$\operatorname{COV}\left(X^{\prime}, X^{\prime \prime}\right)=M\left(X^{\prime} X^{\prime \prime}\right)-M\left(X^{\prime}\right) * M\left(X^{\prime \prime}\right)=$

$p_{12}-p_{1} * p_{2} \quad(1)$

The variances of the vectors $X^{\prime}$ and $X^{\prime \prime}$ are

$$
\sigma\left(X^{\prime}\right)=M\left(X^{\prime}-M\left(X^{\prime}\right)\right)^{2}=M\left(X^{\prime}\right)^{2}-\left(M X^{\prime}\right)^{2}
$$

$\sigma\left(X^{\prime \prime}\right)=M\left(X^{\prime \prime}-M\left(X^{\prime \prime}\right)\right)^{2}=M\left(X^{\prime \prime}\right)^{2}-\left(M X^{\prime \prime}\right)^{2}$

The random variable $\left(X^{\prime}\right)^{2}=X^{\prime} X^{\prime}$ is the intersection of images $X^{\prime}$ and $X^{\prime}$ on the retina and since $\left(X^{\prime}\right)^{2}=X^{\prime}$, then $M\left(X^{\prime}\right)^{2}=M\left(X^{\prime}\right)=p_{1}$ and $\sigma\left(X^{\prime}\right)=M\left(X^{\prime}\right)^{2}-\left(M X^{\prime}\right)^{2}=p_{1}-p_{1}{ }^{2}=$

$p_{1}\left(1-p_{1}\right)=p_{1} * q_{1}, q_{1}=1-p_{1}$

Similarly, since $M\left(X^{\prime \prime}\right)^{2}=M\left(X^{\prime \prime}\right)=p_{2}$ then $\sigma\left(X^{\prime \prime}\right)=M\left(X^{\prime \prime}\right)^{2}-\left(M X^{\prime \prime}\right)^{2}=p_{2}-p_{2}{ }^{2}=$

$p_{2}\left(1-p_{2}\right)=p_{2} * q_{2}, \quad q_{2}=1-p_{2}$

Substituting (1), (2), (3) into the expression for $r_{S}$ we get

$$
r_{s}=\frac{\mathrm{p}_{12}-\mathrm{p}_{1} * \mathrm{p}_{2}}{\sqrt{\mathrm{p}_{1} \mathrm{p}_{2} \mathrm{q}_{1} \mathrm{q}_{2}}}
$$

For random vectors $X^{\prime}$ and $X^{\prime \prime}$, we construct their activity vectors in the associative layer - random variables $\eta^{\prime}$ and $\eta^{\prime \prime}$ and calculate the correlation coefficients between them $[3,6,7]$.

Let $\eta^{\prime}=\left\{\eta_{1}^{\prime}, \eta_{2}^{\prime}, \ldots, \eta_{N_{A}}^{\prime}\right\} \quad$ corresponds to $X^{\prime}=$ $\left\{x_{1}^{\prime}, x_{2}^{\prime}, \ldots, x_{N_{S}}^{\prime}\right\}$, where

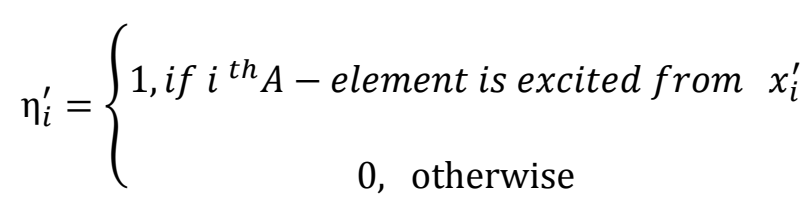
and similarly $\eta^{\prime \prime}=\left\{\eta_{1}^{\prime \prime}, \eta_{2}^{\prime \prime}, \ldots, \eta_{N_{A}}^{\prime \prime}\right\} \quad$ corresponds to $X^{\prime \prime}=\left\{x_{1}^{\prime \prime}, x_{2}^{\prime \prime}, \ldots, x_{N_{S}}^{\prime \prime}\right\}$.Let calculate the correlation coefficient between the random variables $\eta^{\prime}$ and $\eta^{\prime \prime}$

$$
r_{A}=\frac{\operatorname{cov}\left(\eta^{\prime}, \eta^{\prime \prime}\right)}{\sqrt{\sigma\left(\eta^{\prime}\right) \sigma\left(\eta^{\prime \prime}\right)}}
$$

where $\quad \operatorname{COV}\left(\eta^{\prime}, \eta^{\prime \prime}\right)=M\left(\eta^{\prime} \eta^{\prime \prime}\right)-M\left(\eta^{\prime}\right) M\left(\eta^{\prime \prime}\right)$.

We denote $B_{1}$ as the event of exciting an A element on the image $X^{\prime}$. If the event $B_{1}$ occurs, then $\eta^{\prime}$ takes the value 1 , and otherwise - 0 . We denote $B_{2}$ as the event of exciting an A element on the image $X^{\prime \prime}$. If the event $B_{2}$ occurs, then $\eta^{\prime \prime}$ takes the value 1 , and otherwise - 0 . And $B_{3}$ is the event of exciting an A - element on the images $X^{\prime}$ and $X^{\prime \prime}$ (i.e. the events $B_{1}$ and $B_{2}$ occur together). If the event $B_{3}$ occurs , then $\eta^{\prime \prime \prime}$ takes the value 1 , and otherwise 0 .

Let $\eta^{\prime \prime \prime}=\eta^{\prime} \eta^{\prime \prime}=\left\{\eta_{1}^{\prime} \eta_{1}^{\prime \prime}, \eta_{2}^{\prime} \eta_{2}^{\prime \prime}, \ldots, \eta_{N_{A}}^{\prime} \eta_{N_{A}}^{\prime \prime}\right\}$, where

$$
\eta_{i}^{\prime \prime \prime}=\eta_{\mathrm{i}}^{\prime} \eta_{\mathrm{i}}^{\prime \prime}=\left\{\begin{array}{cc}
1, & \text { if } \eta_{\mathrm{i}}^{\prime}=\eta_{\mathrm{i}}^{\prime \prime}=1 \\
0, & \text { otherwise }
\end{array}\right.
$$

As noted above $P_{1}$ and $P_{2}$ are, respectively, the probabilities of excitation of an A-element from $X^{\prime}$ and $X^{\prime \prime}$, and $P_{12}$ the probability of excitation of an A-element from the chain $X^{\prime}$ and $X^{\prime \prime}$, and the covariance between $\eta^{\prime}$ and $\eta^{\prime \prime}$ is

$\operatorname{COV}\left(\eta^{\prime}, \eta^{\prime \prime}\right)=M\left(\eta^{\prime} \eta^{\prime \prime}\right)-M\left(\eta^{\prime}\right) M\left(\eta^{\prime \prime}\right)$

Since the mathematical expectations of $\eta^{\prime}, \eta^{\prime \prime}$ and $\eta^{\prime} \eta^{\prime \prime} \quad$ are

$$
\begin{gathered}
M \eta^{\prime}=1 * P_{1}+0 * Q_{1}=P_{1} \\
M \eta^{\prime \prime}=1 * P_{2}+0 * Q_{2}=P_{2} \\
M\left(\eta^{\prime} \eta^{\prime \prime}\right)=M\left(\eta^{\prime \prime \prime}\right)=1 * P_{12}+0 * Q_{12}=P_{12} \\
Q_{12}=1-P_{12}
\end{gathered}
$$

then substituting them into (4) we get

$$
\operatorname{COV}\left(\eta^{\prime}, \eta^{\prime \prime}\right)=P_{12}-P_{1} P_{2}
$$


To calculate the variances $\sigma\left(\eta^{\prime}\right)$ and $\sigma\left(\eta^{\prime \prime}\right)$ we note that the random variable $\left(\eta^{\prime}\right)^{2}=\eta^{\prime} \eta^{\prime}$ characterizes the intersection of the same set of excited A- elements.

This means that the values assumed by the random variables $\left(\eta^{\prime}\right)^{2}$ and $\eta^{\prime}$ and the probabilities with which they take these values are equal.

The random variable $\left(\eta^{\prime \prime}\right)^{2}$ is defined similarly. Let calculate the variances $\sigma\left(\eta^{\prime}\right)$ and $\sigma\left(\eta^{\prime \prime}\right)$. Since

$$
M \eta^{\prime}=1 * P_{1}+0 * Q_{1}=P_{1}
$$

then

$$
\begin{array}{r}
\sigma\left(\eta^{\prime}\right)=M\left(\eta^{\prime}\right)^{2}-\left(M \eta^{\prime}\right)^{2}=P_{1}-P_{1}^{2}= \\
=P_{1}\left(1-P_{1}\right)=P_{1} Q_{1} .
\end{array}
$$

Similarly,

$$
\begin{gathered}
\sigma\left(\eta^{\prime \prime}\right)=M\left(\eta^{\prime \prime}\right)^{2}-\left(M \eta^{\prime \prime}\right)^{2}=P_{2}-P_{2}^{2}= \\
=P_{2}\left(1-P_{2}\right)=P_{2} Q_{2} .
\end{gathered}
$$

The correlation coefficient between the random variables $\eta^{\prime}$ and $\eta^{\prime \prime}$ will be

$$
r_{A}=\frac{P_{12}-\mathrm{P}_{1} * \mathrm{P}_{2}}{\sqrt{\mathrm{P}_{1} \mathrm{P}_{2} \mathrm{Q}_{1} \mathrm{Q}_{2}}}
$$

Since $r_{S}=r_{A}$ [3], then

$$
\frac{P_{12}-P_{1} * P_{2}}{\sqrt{P_{1} P_{2} Q_{1} Q_{2}}}=\frac{p_{12}-p_{1} * p_{2}}{\sqrt{p_{1} p_{2} q_{1} q_{2}}}
$$

and therefore

$$
P_{12}=\sqrt{\frac{P_{1}}{p_{1}} \frac{P_{2}}{p_{2}} \frac{Q_{1}}{q_{1}} \frac{Q_{2}}{q_{2}}}\left(p_{12}-p_{1} p_{2}\right)+P_{1} P_{2}
$$

The theorem is proved.

Corollary 1. If classes of pathogens satisfy the condition $p_{12}=p_{1} * p_{2}$, then $P_{12}=P_{1} * P_{2}$.

\section{Proof.}

Let the pathogens $\mathrm{X}^{\prime}$ and $\mathrm{X}^{\prime \prime}$ excite the retina with propabilities $\mathrm{p}_{1}$ and $\mathrm{p}_{2}$ respectively. The condition $p_{12}=p_{1} * p_{2} \quad$ means that these random variables are independent. It follows from the formula (5) that $\mathrm{P}_{12}=\mathrm{P}_{1} * \mathrm{P}_{2}$, i.e. in the associative layer, the independence of the random variables $\eta^{\prime}$ and $\eta^{\prime \prime}$ is preserved.

This result coincides with the result of Theorem 4 given in [3, p. 306], where the proof is presented in a completely different way.
Corollary 2. When the intersection area of two images on the retina decreases, then the efficiency of the neural network increases (that is, the degree of correct recognition increases).

\section{Proof.}

When an image appears on the retina, then an Aelement is either excited or remains not excited.

For each class of pathogens S.V Dayan introduced the characteristic function (CHF) of the perceptrontype neural network as a measure of the quality of recognition [3]. In the case of two classes, this function has the form

$$
\zeta_{\mathrm{i}}=P_{\mathrm{i}}-P_{12} \quad,(\mathrm{i}=1,2),
$$

where $\quad P_{i}(i=1,2)$ - is the probability that an Aelement is excited when a single image appears from the i-th class, and $P_{12}$ is the probability of exciting an A-element from pathogens from both classes.

Note that the CHF characterizes the probability that the A-element is excited when a pathogen belonging to a certain class is shown and is not excited by a pathogen not belonging to this class.

If $p_{12} \rightarrow 0$, then, as proved in [3], $P_{12} \rightarrow 0$. Thus, when $p_{12} \rightarrow 0$, that is, when the intersection area of two images on the retina decreases, $\zeta_{1}$ and $\zeta_{2}$ tend to their maximum values. This means that the correct recognition for stimuli of the first and second class tends to the maximum.

The obtained dependence (5) is a convenient mathematical tool for the study of the statistical characteristics of neural networks and for the synthesis of their effective structures.

\section{Conclusion}

The probabilistic methods for the study of neural networks allow to obtain its optimal parameters in order to improve its classification ability. The results obtained in the proposed paper contribute to the solution of such problems. They are also interesting because they are consistent with the results obtained by a different path for the perceptron-type neural network. 
References:

[1] Frank Rosenblatt F. Principles of Neurodynamics. Perceptrons and Theory of Brain Mechanisms. M., Mir, 1965, (in Russian)

[2] Perceptron - pattern recognition system, Edited by Ivahnenko A.G., Kiev, Naukova Dumka, 1975, (in Russian).

[3] Kroug P.G. Neural Networks and Neurocomputers: Course Tutorial "Microprocessors". - M .: Publishing house MEI, 2002, (in Russian)

\section{Contribution of individual authors to the creation of a scientific article}

Siranush Sargsyan has given the main idea, created the model, proved the main theorem.

Anna Hovakimyan has proved the corollaries and prepared the text.
[4] Sevastyanov B.A. The course of probability theory and mathematical statistics, M., Nauka, 1982.

[5] S. Sargsyan, A. Hovakimyan, Probabilistic Methods for Neural Networks Study, Quarterly Journal of Mechanics and Applied Mathematics, Issue 4 (2), Volume 69,“Oxford University Press”, Nov. 2016, pp.669-675

[6] S. Sargsyan, A. Hovakimyan, Statistical Evaluation of the Performance of the Neural Network, London Journal of Research in Computer Science and Technology, Volume 17 | Issue 1 | Compilation 1.0, 2017, pp.1-6

\section{Creative Commons Attribution License 4.0 (Attribution 4.0 International, CC BY 4.0)}

This article is published under the terms of the Creative Commons Attribution License 4.0

https://creativecommons.org/licenses/by/4.0/deed.en_US 